\title{
Factores de riesgo que influyen en el fracaso al tratamiento individualizado de tuberculosis multidrogorresistente en la provincia de Ica. 2000-2014.
}

\author{
Risk factors that influence in the failure to the individualized \\ treatment of multidrug-resistant tuberculosis in the province of ica. \\ 2000-2014.
}

del Rio-Mendoza Jeny', del Rio-Mendoza Jesús Antonio', Gómez-Donayre Marth ysabel', Buleje-Medina Fiorella ${ }^{1}$, Montalvo-Valdez Hugo Ángel ${ }^{1}$

1. Universidad Nacional San Luis Gonzaga de Ica.

a. Especialista en Medicina Interna- Doctor en Salud Pública.

b. Médico Cirujano.

c. Lic. En Enfermería.

d. Especialidad Anestesiología - Magister en Medicina Humana.

\section{Correspondencia:}

Jeny Rosanna Julia del Rio-Mendoza Profesión: Médico Cirujano. Especialidad Medicina Interna.

Número de celular: 956622171 Correo Electrónico:

jdelriom7@gmail.com

Domicilio: Ica, Perú.

Contribuciones De Autoría: dRMJ, dRMJA, GDMY, BMF y MVHA: Contribuciones en la concepción y diseño del manuscrito, recolección, análisis e interpretación de los datos, redacción y revisión crítica del contenido del manuscrito y aprobación final del artículo.

Conflicto De Intereses: No declarados.

Financiamiento: Autofinanciado.

\section{Como Citar}

del Rio-Mendoza Jeny, del Rio-Mendoza Jesús Antonio, Gómez-Donayre Marth ysabel, Buleje-Medina Fiorella, Montalvo-Valdez Hugo Ángel. Factores de riesgo que influyen en el fracaso al tratamiento individualizado de tuberculosis multidrogorresistente en la provincia de Ica. 2000-2014. Rev méd panacea 2018; (3): 83-88

Recibido: 26 - 09 - 2018

Aceptado: 30 - 11 - 2018

Publicado: 28 - 12 - 2018

\section{RESUMEN}

Objetivo: Determinar los factores de riesgo que influyen en el fracaso al tratamiento individualizado de Tuberculosis Multidrogorresistente en la provincia de Ica. 2000-2014. Materiales y métodos: Estudio observacional, retrospectivo, tipo analítico, de casos y controles. La muestra estuvo conformada por 19 casos de fracaso al tratamiento individualizado, y por cada caso se tuvo dos controles de pacientes que curaron con tratamiento individualizado. Se realizó el análisis bivariado con un nivel de significancia del $5 \%$ se calculó del Odds Ratio (OR) con intervalo de confianza (IC) al 95\%. Se realizó análisis de regresión logística. Resultados: En el análisis multivariado resultaron 4 factores de riesgo asociados a fracaso al tratamiento individualizado: resistencia a 5 o más drogas $(\mathrm{OR}=6,67, \mathrm{p}=0,027)$, tener $\mathrm{IMC}$ menor de 18.5 al inicio del tratamiento $(\mathrm{OR}=7,61 \mathrm{p}=0,023)$, presentar hemoptisis durante el tratamiento $(\mathrm{OR}=19,89, \mathrm{P}=0,001)$ y la presencia de cavernas en la radiografía de tórax inicial $(O R=27,95, p=0,005)$. Conclusiones: Los pacientes con resistencia a 5 o más drogas antituberculosas, con IMC menor a 18.5, con hemoptisis durante el tratamiento y los que presentan caverna en la radiografía de tórax, tienen mayor riesgo de fracasar al tratamiento individualizado.

Palabras clave: Tuberculosis, Multidrogorresistente, factores de riesgo.

\section{ABSTRACT}

Objective: To determine the risk factors that influence the failure to individualized treatment of multidrug-resistant tuberculosis in the province of Ica. 2000-2014. Materials and methods: Observational, retrospective study, analytical type, of cases and controls. The sample consisted of 19 cases of failure to individualized treatment, and for each case there were two controls of patients who cured with individualized treatment. The bivariate analysis was performed with a level of significance of $5 \%$ was calculated from the Odds Ratio (OR) with $95 \%$ confidence interval $(\mathrm{Cl})$. Logistic regression analysis was performed. Results: In the multivariate analysis, there were 4 risk factors associated with failure to individualized treatment: resistance to 5 or more drugs $(O R=6.67$, $p=0.027)$, having a BMI less than 18.5 at the beginning of treatment $(O R=7,61 p=0.023)$, presenting hemoptysis during treatment $(O R=19.89, P=0.001)$ and the presence of caverns on the initial chest radiograph $(O R=27.95, p=0.005)$. Conclusions: Patients with resistance to 5 or more antituberculous drugs, with a BMI less than 18.5, with hemoptysis during treatment and those who have a cavern on chest radiography, have a higher risk of failing individualized treatment.

Keywords: Tuberculosis, Multidrug-resistant, risk factors. 


\section{INTRODUCCIÓN}

La Tuberculosis multidrogorresistente (TB MDR) y Tuberculosis extremadamente resistente (TB XDR), constituyen uno de los problemas de salud pública más importantes en el país (1). Está vinculada a la pobreza, malas condiciones de trabajo y vida (2). Ha experimentado un dramático revés con la aparición de resistencia a los tratamientos habituales (3), y es una amenaza emergente para el control mundial (4).

La Tuberculosis MDR es causada resistencia a isoniazida y rifampicina. TB XDR es la TB MDR más resistencia a una fluoroquinolona y al menos a una droga inyectable (5) (6), y Tuberculosis totalmente resistente (TB-TR), muestra resistencia a todos los fármacos (7).

El antecedente de tratamiento y abandono incrementan el riesgo de TB MDR (8). La mejor opción de manejo es el tratamiento individualizado, basado en la prueba de sensibilidad (9). El tratamiento se complica y el pronóstico empeora a medida que hay mayor resistencia (7). La eficacia de los fármacos de segunda línea es menor, el tiempo de tratamiento es mayor, y tiene más efectos adversos (4). La tasa de curación varía, con eficacia mayor al $90 \%$ en algunos estudios, mientras que en otros no cura ni al 50\% (6). Hay fracaso cuando no hay conversión bacteriológica al sexto mes o cuando hay reversión bacteriológica después del sexto mes. También cuando hay ampliación de resistencia, a una fluoroquinolona y un inyectable de segunda línea (10). Predicen fracaso al tratamiento: Historia de terapia previa, mayor cantidad de medicamentos resistentes, cavidades, cultivos positivos después de 2 a 3 meses de tratamiento y comorbilidad por VIH (11). Además, el índice de masa corporal (IMC) y el abuso de alcohol se asocian a resultado desfavorable (12).

El tratamiento individualizado representa la última oportunidad de cura del paciente (13). La mayoría de las investigaciones han usado esquemas con 4 a 6 medicamentos (11). Debe incluir un inyectable, una fluoroquinolona, dos drogas de primera línea, o en su defecto de segunda línea, y que al menos cuatro fármacos con eficacia confirmada (14). El inyectable se debe usar, hasta contar con 4 cultivos negativos y un máximo de 6 a 8 meses (10). Dura dos años o más por lo que se necesita un indicador que permita evaluar si al paciente va ir bien antes de finalizar el tratamiento (15). Es importante conocer los factores que influyen en el fracaso al tratamiento, para así detectar en forma temprana aquellos pacientes en riesgo.

El objetivo de este estudio es determinar los factores de riesgo que influyen en el fracaso al tratamiento individualizado de Tuberculosis Multidrogorresistente en la provincia de Ica.

\section{MATERIALES Y MÉTODOS}

Estudio observacional, retrospectivo, analítico, de casos y controles. La población estuvo conformada por los casos de Tuberculosis pulmonar MDR que recibieron tratamiento individualizado en la Provincia de Ica entre los años 2000 y 2014, y fueron registrados en el Comité Regional de Evaluación de retratamiento (CRER), del año 2000 hasta el 2014. La muestra estuvo constituida por 19 casos, y 38 controles. Los criterios de inclusión de los casos incluyeron pacientes con TB MDR que fracasaron al tratamiento individualizado. Criterios de inclusión de los controles: TB MDR curados con el esquema individualizado. Criterios de exclusión: menores de 15 años y Tuberculosis extrapulmonar. Se revisó las Historias Clínicas, tarjetas de tratamiento y actas de los pacientes, en los archivos de la Estrategia Regional de Tuberculosis y en los establecimientos de salud. Se elaboró una ficha de recolección de datos. Las variables consignadas en la ficha, fueron consideradas teniendo en cuenta los antecedentes de la investigación, sin embargo no se incluyeron otras variables importantes como factores de riesgo, debido a que por ser un estudio retrospectivo los datos son limitados en la historia clínica. Se creó una base de datos en el programa estadístico SPSS v. 22 para ingresar las fichas y realizar el análisis. Se utilizó técnicas estadísticas univariadas, bivariadas y multivariadas. Se realizó análisis estadístico con $x^{2}$, Test exacto de Fisher, ODSS RATIO, valor $p, I C$ al $95 \%$.

\section{RESULTADOS:}

De acuerdo a los grupos etarios, el mayor porcentaje de pacientes se encontró en el grupo de 20 a 32 años (45.6\%), seguido por el grupo de menores de 20 años (21.1\%), y el grupo de 33-44 años (17.5\%). No hubo diferencia significativa en los datos sociodemográficos en el grupo de casos respecto a los controles como se muestra en la Tabla 1.

Tabla 1: La edad y su asociación con la aparición de cáncer de cuello uterino.

\begin{tabular}{|c|c|c|c|}
\hline Variable & Casos & Controles & $\mathbf{p}$ \\
\hline Edad (media) & 30,2 & 30,6 & 0,44 \\
\hline Sexo & & & 0,09 \\
\hline Masculino & $7(36,8 \%)$ & $23(60,5 \%)$ & \\
\hline Femenino & $12(63,2 \%)$ & $15(39,5 \%)$ & \\
\hline Ocupación & & & 0,39 \\
\hline Estudiante & $2(10,5 \%)$ & $9(23,7 \%)$ & \\
\hline Ama de casa & $9(47,4 \%)$ & $8(21,1 \%)$ & \\
\hline Obrero & $2(10,5 \%)$ & $4(10,5 \%)$ & \\
\hline Comerciante & $0\left(\begin{array}{ll}0 & \%\end{array}\right)$ & $1(2,6 \%)$ & \\
\hline No trabaja & $3(15,8 \%)$ & $10(26,3 \%)$ & \\
\hline Otros & $3(15,8 \%)$ & $6(15,8 \%)$ & \\
\hline Grado de Instrucción & & & 0,06 \\
\hline Primaria & $5(26,3 \%)$ & $3(7,9 \%)$ & \\
\hline Secundaria & $11(57,9 \%)$ & $23(60,5 \%)$ & \\
\hline Técnico & $1(5,3 \%)$ & $0(0,0 \%)$ & \\
\hline Superior & $2(10,5 \%)$ & $12(31,6 \%)$ & \\
\hline Estado civil & & & 0,89 \\
\hline Casado & $2(10,5 \%)$ & $3(7,9 \%)$ & \\
\hline Soltero & $11(57,9 \%)$ & $17(44,7 \%)$ & \\
\hline Conviviente & $4(21,1 \%)$ & $12(31,6 \%)$ & \\
\hline Viudo & $1(5,3 \%)$ & $3(7,9 \%)$ & \\
\hline Separado/divorciado & $0(0 \%)$ & $1(2,6 \%)$ & \\
\hline No datos & $1(5,3 \%)$ & $2(5,3 \%)$ & \\
\hline
\end{tabular}


Se realizó el análisis bivariado, con las variables independientes, teniendo en cuenta que la variable severidad de la enfermedad engloba presencia de síntomas agudos (disnea, hemoptisis, fiebre), Índice de masa corporal $(\mathrm{IMC})<18.5$, resistencia a 5 o más drogas, y presencia de cavernas en la radiografía; la variable deterioro clínico incluye disminución de peso durante el tratamiento y hemoptisis. Se encontró trece variables como factores de riesgo, como se muestra en la Tabla 2.

Tabla 2: Factores de riesgo que influyen en el fracaso al tratamiento individualizado de Tuberculosis multidrogorresistente en la provincia de Ica. 2000-2014, en el análisis bivariado.

\begin{tabular}{|c|c|c|c|c|}
\hline VARIABLE & OR & & C 95\% & \\
\hline Comorbilidad & 0,65 & 0,06 & $-6,68$ & 1 \\
\hline Antecedente de Tratamiento & 1,28 & 0,22 & $-7,35$ & 1 \\
\hline Severidad de enfermedad & 14,57 & 1,76 & $-120,52$ & 0 \\
\hline Síntomas agudos & 44,18 & 5,2 & $-\quad 372,6$ & 0 \\
\hline Disnea inicial & 10,5 & 2,4 & $-\quad 46,3$ & 0 \\
\hline Hemoptisis inicial & 20 & 4,5 & $-\quad 89,9$ & 0 \\
\hline Fiebre & 1,5 & 0,32 & - $\quad 7,98$ & 0,68 \\
\hline $\mathrm{IMC}<18.5$ & 6,09 & 1,8 & $-\quad 20,7$ & 0 \\
\hline Resistencia a 5 o más drogas & 7,59 & 2,2 & $-\quad 26,3$ & 0 \\
\hline Cavernas en la radiografía & 39 & 4,7 & $-327,1$ & 0 \\
\hline Demora en iniciar Tratamiento & 4,8 & 1,42 & $-\quad 16,18$ & 0,01 \\
\hline Uso de esquemas no eficaces & 4,06 & 0,46 & $-\quad 35,75$ & 0,25 \\
\hline Demora en conversión del esputo & 20 & 3,71 & $-107,82$ & 0 \\
\hline Deterioro clínico & 153 & 19,83 & $-1180,28$ & 0 \\
\hline Hemoptisis durante tratamiento & 50,88 & 5,7 & $-452,3$ & 0 \\
\hline Disminución de peso & 197,33 & 19,0 & $-2044,3$ & 0 \\
\hline Extensión radiológica moderada & 16,6 & 4,2 & $\begin{array}{l}-\quad 65,7 \\
-\end{array}$ & 0 \\
\hline
\end{tabular}

Las variables que se asociaron significativamente a riesgo de fracaso al tratamiento individualizado en el análisis bivariado, se analizaron mediante regresión logística multivariada, apreciándose 4 variables que se asociaron a riesgo de fracaso, como se muestra en la Tabla 3.

Tabla 3: Factores de riesgo que influyen en el fracaso al tratamiento individualizado de Tuberculosis multidrogorresistente en la provincia de Ica. 2000-2014: Variables asociadas independientemente en el análisis multivariado.

\begin{tabular}{lccc}
\multicolumn{1}{c}{ VARIABLE } & OR & IC 95\% & Valor $\mathbf{p}$ \\
Resistencia a 5 o más drogas & 6,67 & $1,24-35,92$ & 0,027 \\
IMC menor de 18,5 & 7,61 & $1,33-43,58$ & 0,023 \\
Presencia de cavernas & 27,95 & $2,75-284,49$ & 0,005 \\
Hemoptisis en el tratamiento & 19,89 & $1,49-265,48$ & 0,001 \\
\hline
\end{tabular}

\section{DISCUSIÓN}

En la presente investigación la variable comorbilidad no se halló como un factor de riesgo de fracaso al tratamiento, a diferencia de la investigación realizada por Hye, quien comparó dos grupos, de éxito y fracaso al tratamiento de TB MDR, encontrando que la presencia de comorbilidad fue factor de riesgo independiente para predecir fracaso al tratamiento individualizado (OR ajustado, 2.62, IC 95\%:1.00-6.87) (16). Igualmente, Ahmad, en una cohorte de 173 pacientes, encontró que 
la comorbilidad fue factor de riesgo de tratamiento no exitoso (OR: 3.785, IC 95\%:1.201-11.922, $p=0.023)(17)$. Samuels por el contrario investigó la asociación de fracaso al tratamiento y comorbilidades, encontrando asociación con VIH (RR: 1,4) y consumo de alcohol (RR: $1,45)$, no encontrando asociación con Diabetes ni enfermedad renal (18).

El haber recibido drogas antituberculosas en tratamientos anteriores no se halló como factor de riesgo en esta investigación, a diferencia del estudio realizado por Lemaine, quien encontró asociación independiente con resultado pobre al tratamiento individualizado, teniendo seis veces más probabilidad de tener resultado pobre al tratamiento que aquellos que nunca recibieron tratamiento para tuberculosis (19). Brust igualmente, en un estudio retrospectivo, encontró que el haber recibido tratamiento anterior de tuberculosis, se asoció a fracaso a tratamiento individualizado (aOR 1.7. IC: 1.0-2.8) (20). Goble en su investigación de 134 pacientes en tratamiento individualizado, encontró en el análisis multivariado, que la administración previa de 8-11 drogas (OR: 4. IC 95\%: 1. 6- 9.9) fue asociada significativamente con fracaso a tratamiento $(p<0.001)$ y no se encontró asociación a uso previo de 2-5 drogas ni 6-7 drogas (21). Martínez igualmente encontró asociación con fracaso el recibir 8-11 drogas en tratamientos anteriores (22). Johnston encontró que el no tener tratamiento previo fue asociado a tratamiento exitoso 1.42 [1.05-1.94] (12).

La presencia de alguna condición de severidad de la enfermedad no fue un factor de riesgo en el análisis multivariado. Sin embargo al realizar el análisis de los factores que dan la severidad de la enfermedad, en forma individual, solo la resistencia a 5 o más drogas y el tener IMC < 18.5 se hallaron como factores de riesgo de fracaso al tratamiento individualizado en el análisis multivariado. El tener resistencia a 5 o más drogas (OR=6,67, IC 95\%: 1,24-35-92; $p=0,027)$, incrementó en 6 veces más el riesgo de fracaso, coincidiendo con Martínez, quien en una revisión de publicaciones agrupó población con resultado favorable al tratamiento en 33 a $66 \%$, encontrando que la resistencia a 5 fármacos o más se asoció a pobre resultado al tratamiento (22). Brust en un estudio retrospectivo de pacientes con TBC MDR, realizó el análisis multivariado, encontrando como factores de riesgo independientes de fracaso al tratamiento, el tener resistencia a 3 fármacos (aOR 2.3. IC: 1.3-4.1) y resistencia a 5 fármacos (OR 3.0. IC: 1.5-5.7) (20). Esta investigación difiere con la investigación realizada por Lemaine, quien no encontró asociación con fracaso al tratamiento y el tener resistencia a cinco o más drogas en el análisis multivariado (19). Johnston tampoco encontró asociación significativa el tener resistencia a 6 drogas o más con un resultado pobre al tratamiento (12). Si bien es cierto que el encontrar ya resistencia amplia a los medicamentos, es un factor de riesgo que no lo podemos modificar, sí sería necesario tomarlo muy en cuenta para la elaboración de esquemas adecuados, desde el inicio, tomando en cuenta los resultados de la prueba de sensibilidad convencional y tomando en cuenta la eficacia de los medicamentos, interrogando a los pacientes sobre los medicamentos recibidos en tratamientos anteriores y realizando a la vez un monitoreo estricto multidisciplinario, que nos permita evaluar la eficacia del tratamiento, ya que estaríamos en riesgo no solamente de no curar, sino además de generar más resistencia a fármacos, incrementando el número de medicamentos resistentes, y perpetuar la cadena de contagio a los contactos, incrementando el número de casos de TB MDR.

El tener IMC menor de 18.5 al inicio del tratamiento fue 7 veces mayor riesgo de fracaso en el análisis multivariado en esta investigación ( $O R=7,61 p=0,023)$, coincidiendo con la investigación realizada por Lemaine en 178 pacientes, quien encontró asociación independiente con fracaso al tratamiento $(O R=2.3$, 1.1-4.9, $p=0.03$ ) (19). Igualmente Johnston en una revisión sistemática de 8 estudios encontró asociación con un IMC bajo (12). Martínez igualmente encontró asociación con IMC < 18.5 (22). Este es un factor que debemos tenerlo muy en cuenta, desde el inicio del tratamiento, ya que puede modificarse en el menor tiempo, poniendo énfasis desde el inicio en el aspecto nutricional del paciente y haciendo un seguimiento permanente para asegurar un resultado favorable, mejorando el estado nutricional, a fin de disminuir el riesgo de fracaso al tratamiento. La demora en iniciar el tratamiento desde la sospecha del diagnóstico hasta el inicio del tratamiento y el uso de esquemas no eficaces, no fueron factores de riesgo de fracaso al tratamiento en esta investigación.

La demora en la conversión del esputo al tercer mes no se encontró como factor de riesgo de fracaso al tratamiento individualizado, a diferencia de Kurbatova quien estudió 1768 pacientes, encontrando como factor de riesgo para fracaso al tratamiento de TB MDR en el análisis multivariado, la no conversión de cultivo al tercer mes (RR = 5.84 (3.02-11.27)) (23). Atif en su investigación encontró asociación con fracaso el tener cultivo + al segundo mes de tratamiento (24).

Respecto al deterioro clínico, no se halló como factor de riesgo de fracaso en esta investigación, sin embargo la hemoptisis fue factor de riesgo en el análisis multivariado $(O R=19.89 p=0,001)$.

La presencia de cavernas en la radiografía de tórax, fue un factor 28 veces de riesgo de fracaso al tratamiento $(\mathrm{OR}=27,95, \mathrm{p}=0,005)$, coincidiendo con Ahmad quien en una cohorte en 181 pacientes con TB MDR, encontró en el análisis de regresión logística como factor de riesgo la presencia de cavernas en la radiografía de tórax (OR: 5.25, IC 95\%:2.26-12.22, $p=0,001$ ) (17). Martínez, igualmente encontró, que la presencia de cavitaciones en la radiografía de tórax se asoció a resultado desfavorable al tratamiento (22). Igualmente, Yew, en un estudio de 79 pacientes con TB MDR encontró que la presencia de cavidad en la radiografía, se asoció a fracaso al tratamiento o muerte (OR: 15.9 IC: 1.9-135.3) (25). Hye encontró que la presencia de cavidades 
bilaterales predecía el fracaso del tratamiento (OR ajustado, 2.42, IC: 0.95-6,19, p: 0,06 (16). Por el contrario, Johnston en una revisión sistemática no encontró asociación significativa con la presencia de cavernas $(\mathrm{OR}=0,62,0,28-1,36)(12)$. Es necesario tener en cuenta que la presencia de cavernas puede influir disminuyendo la penetración de los medicamentos y por lo tanto atenuar la eficacia de los fármacos antituberculosos, por lo cual los pacientes deben ser evaluados, con radiografía de tórax buscando presencia de cavernas y hacer un seguimiento estricto.

De los 4 factores de riesgo encontrados en esta investigación en el análisis multivariado, el que tuvo mayor fuerza de asociación fue la presencia de cavernas, seguido de hemoptisis durante el tratamiento, sin embargo es necesario recalcar que los intervalos de confianza son muy amplios, lo cual podría deberse al tamaño de la muestra, hay que considerar que los establecimientos de salud periféricos muchas veces no cuentan con estudios de imagen, lo cual dificulta la evaluación adecuada de los pacientes. Igualmente es necesario interrogar durante la evaluación médica, sobre presencia de hemoptisis, ya que siendo un factor de

\section{REFERENCIAS BIBLIOGRÁFICAS}

1. Ministerio de Salud del Perú. Comité de Tuberculosis Extremadamente Resistente (TB XDR). Situación actual y propuesta de lineamientos técnicos para el control de la tuberculosis resistente en el Perú. Lima: Ministerio de Salud; 2008.

2. Fuentes L. Enfoque sociopolítico para el control de la tuberculosis en el Perú. Rev Peru Med Exp Salud Pública. 2009; 26 (3): 370-79.

3. Lado Lado F. L., García Ramos R, Pérez del Molino ML, Ferreiro Regueiro MJ, Maceda Vilariño $S$, Túnez Bastida V. Tuberculosis resistente a fármacos. An Med Interna [Internet] 2004. [citado 22 jul 2016]; 21(4):46-52. Disponible en: http://scielo.isciii.es/scielo.php?script=sci_arttext\&pid =S0212-71992004000400010

4. Secretaría de salud de México. Guía para la Atención de Personas con Tuberculosis Resistente a Fármacos. México: Secretaría de Salud; 2010.

5. World Health Organization. Multidrug and extensively drug-resistant TB (M/XDR-TB) 2010. Global Report on Surveillance and response. World Health Organization. 2010. [citado 21 Jul 2016]. Disponible en:

http://whqlibdoc.who.int/publications/2010/9789241 599191_eng.pdf

6. Ministerio de salud del Perú. Informe de gestión: Estrategia Sanitaria Nacional de prevención y control de Tuberculosis: 2001-2006. Lima: Ministerio de Salud; 2006.

7. Caminero JA. Tratamiento de la tuberculosis según el diferente patrón de resistencias. Med Clin (Barc). 2010; 134(4):173-181. riesgo, podrían los pacientes fracasar al tratamiento. Por lo tanto, es importante hacer una evaluación correcta en el paciente con diagnóstico de TB MDR, detectando en forma temprana aquellos factores que pueden influir y constituirse en riesgo de fracaso al tratamiento individualizado, para así enfocarnos y elaborar esquemas de tratamiento más eficaces con drogas antituberculosas que aseguren un resultado positivo, así como tener una evaluación permanente sobre la evolución de estos pacientes, y detectar en forma temprana aquellos factores que pueden ser riesgo de fracaso al tratamiento individualizado. En esta investigación de los cuatro factores de riesgo encontrados, 3 se presentan al inicio de la enfermedad, cuando se capta a los pacientes, y uno de ellos es modificable, el IMC, mientras que el otro factor que es hemoptisis se evalúa durante el tratamiento, debiendo interrogar bien a los pacientes al ser un factor de riesgo de fracaso.

Esta investigación tuvo limitaciones, en el sentido de los datos que fueron tomados de historias clínicas, y por ser retrospectivo no se registró datos completos en todas las historias.

8. del Rio-Mendoza J, Guillén G, Torres J, del Rio J. Factores de riesgo para Tuberculosis multidrogorresistente en la provincia de Ica. Dirección regional de salud. Ica. 2000-2002. Enfermedades del Tórax. Revista de la Sociedad Peruana de Neumología. 2005; 49 (3).

9. Ministerio de Salud del Perú. Norma Técnica de salud para el control de la Tuberculosis. Dirección General de Salud de las Personas. Lima: Ministerio de Salud; 2006.

10. Ministerio de Salud del Perú. Norma técnica de salud para la atención integral de las personas afectadas por Tuberculosis. Lima: Ministerio de Salud; 2013.

11. Southeastern National Tuberculosis Center. Tuberculosis Fármacorresistente: Una Guía Práctica para la Atención Médica del Paciente. [internet] 2009. [citado 21 Jul 2016] Disponible en: http://docplayer.es/4641763-Tuberculosis-farmacor resistente-una-guia-practica-para-la-atencion-medi ca-del-paciente.html

12. Johnston JC, Shahidi NC, Sadatsafavi M, Fitzgerald JM. Treatment Outcomes of Multidrug-Resistant Tuberculosis: A Systematic Review and Meta-Analysis. PLoS One. 2009; 4(9):6914.

13. Ministerio de Salud del Perú. Construyendo alianzas estratégicas para detener la Tuberculosis: La experiencia peruana. Lima: Ministerio de salud; 2006.

14. Mendoza A, Gotuzzo E. Tuberculosis extremadamente resistente (TB-XDR): historia $y$ situación actual. Acta méd. Peruana. 2008; 25(4):236-46. 
15. World Health Organization. Multidrug- resistant tuberculosis (MDR-TB) Indicators. A minimum set of indicators for the programmatic management of MDR-TB in national tuberculosis control programmes. World Health Organization [internet] 2010. [citado 12 Ag 2012]. Disponible en: http://whqlibdoc.who.int/hq/2010/WHO_HTM_TB_20 10.11_eng.pdf

16. Hye-Ryoun Kim, Seung Hwang, Hyun Kim, Sang Lee, Chul-Gyu Yoo, Young Kim, Sung Han, Young-Soo Shim, Jae-Joon Yim. Impacto de la resistencia farmacológica extensa en los resultados del tratamiento en pacientes no infectados por $\mathrm{VIH}$ con tuberculosis multirresistente. Clin Infect Dis [internet] 2007. [citado 12 Ag 2016]; 45 (10):1290-1295. Disponible

en: https://academic.oup.com/cid/article/45/10/1290/27 7486

17. Ahmad N, Javaid A, Basit A, Afridi A, Khan M, Ahmad L, Sulaiman $S$, Khan A. Management and treatment outcomes of MDR-TB: results from a setting with high rates of drug resistance. Int. J. Tuberc Lung Dis [internet] 2015. [citado 12 Ag 2016]; 19(9):1109-1114. Se consigue en: http://www.ingentaconnect.com/content/iuatld/ijtld/ 2015/00000019/00000009/art00021\#expand/collaps e

18. Samuels JP, Sood A, Campbell JR, Ahmad Khan F, Johnston JC. Comorbidities and treatment outcomes in multidrug resistente tuberculosis: a systematic review and meta-analysis. Sci Rep [internet] 2018. [citado 12 Ag 2016]; 8: 4980. Se consigue en: https://www.nature.com/articles/s41598-018-23344-z

19. Leimane V, Riekstina V, Holtz T, Zarovska $E$, Skripconoka V, Thorpe L, Laserson K, Wells Ch. Clinical outcome of individualised treatment of multidrug-resistant tuberculosis in Latvia: a retrospective cohort study. Lancet. 2005; 365:318- 26.
20. Brust J, Gandhi Neel, Carrara H, Osburn G, Padayatchi N. Treatment Failure and Default Rates for Patients with MDR-TB in KwaZulu- Natal, South Africa, 2000-2003. Int J Tuberc Lung Dis [internet] 2010 [Citado 11 abril 2014]; 14(4): 413-419. Disponible en: http://www.ncbi.nlm.nih.gov/pmc/articles/PMC3005 763/pdf/nihms254519.pdf

21. Goble $M$, Iseman $M$, Lorie A, Waite $D$, Ackerson $L$, Horsburgh R. Treatment of 171 patients with pulmonary tuberculosis resistant to isonizid and rifampin. N Engl J Med. 1993; 328(8): 527-32.

22. Martínez $D$, López $E$, Torres $A$, Ruiz $V$, Mora $M$, Salazar M. Consideraciones a los resultados de tratamiento en pacientes con tuberculosis resistente a múltiples fármacos (TB MDR). Una revisión de la literatura. Neumol Cir Torax. 2008; 67(1):34-43.

23. Kurbatova E, Taylor A, Gammino V, Bayona J, Becerra M, Danilovitz M, Falzon D, Gelmanova I, Keshavjee S, Leimane V, Mitnick C, Quelapio M, Riekstina V, Viiklepp P, Zignol M, Cegielski P. Predictors of poor outcomes among patients treated for multidrug-resistant tuberculosis in DOTS-plus projects. Tuberculosis (Edinb). 2012; 92 (5) 388-396.

24. Atif M, Bahir A, Ahmad N, Kaneez R, Saba S, Scahill S. Predictors of unsuccessful interim treatment outcomes of multidrug resistant tuberculosis patients. BMC Infect Dis [internet] 2017. [citado Dic 2017]; 17(1):655. Se consigue en: https://www.ncbi.nlm.nih.gov/pmc/articles/PMC562 2487/

25. Yew W, Chan K, Chau C, Tam CM, Leung C, Leung C, Wong $\mathrm{P}$, Lee J. Outcomes of Patients With Multidrug-Resistant Pulmonary Tuberculosis Treated With Ofloxacin/ Levofloxacin-Containing Regimens. Chest. 2000; 117(3):744-51

\section{Las ediciones anteriores de revista médica PANACEA están disponibles en:}

\section{www.revpanacea.unica.edu.pe}

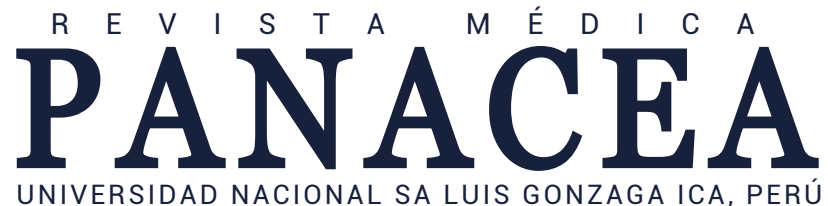

